Marquette University

e-Publications@Marquette

Biomedical Engineering Faculty Research and

Publications

Biomedical Engineering, Department of

$1-1-2002$

\title{
A Pneumatically Actuated Manipulandum for Neuromotor Control Research
}

\author{
Aaron J Suminski \\ Marquette University \\ Kristina M. Ropella \\ Marquette University, kristina.ropella@marquette.edu \\ Robert A. Scheidt \\ Marquette University, robert.scheidt@marquette.edu
}

Accepted version. Published as part of the proceedings of the conference, 24th Annual Conference and the Annual Fall Meeting of the Biomedical Engineering Society EMBS/BMES Conference, 2002:

2347-2348. DOI. (C) 2002 Institute of Electrical and Electronics Engineers (IEEE). Used with permission. 


\title{
A pneumatically Actuated Manipulandum For Neuromotor Control Research
}

\author{
A.J. Suminski \\ Department of Biomedical Engineering, Marquette University, Milwaukee, WI \\ K.M. Ropella \\ Department of Biomedical Engineering, Marquette University, Milwaukee, WI \\ R.A. Scheidt \\ Department of Biomedical Engineering, Marquette University, Milwaukee, WI
}

\begin{abstract}
Functional magnetic resonance imaging (fMRI) techniques have great potential for identifying which neural structures are involved in the control of goal-directed reaching movements. However, fMRI techniques alone are not capable of probing the neural mechanisms involved in acquisition of novel motor behaviors because such studies require that the moving limb be perturbed in a controlled fashion. We outline a plan to design and develop a non-metallic, pneumatically actuated tool that, along with systems identification techniques and functional magnetic resonance imaging (fMRI), will characterize and quantify how the human central nervous system uses sensory information during practice-based motor learning.
\end{abstract}


NOT THE PUBLISHED VERSION; this is the author's final, peer-reviewed manuscript. The published version may be accessed by following the link in the citation at the bottom of the page.

\section{SECTION I.}

\section{Introduction}

Many regions of the brain are thought to be involved in the acquisition and control of goal-directed movements, including the supplemental motor, premotor and motor cortices, basal ganglia and cerebellum. ${ }^{1}$ While it is possible to study the activity of neural structures during reaching in awake, behaving animals with microelectrodes, ${ }^{2}$ it is obviously not possible to do so in humans. Instead, indirect measures of neural activity, such as functional magnetic resonance imaging (fMRI), have been used to study neural activity related to a variety of motor-related behaviors including isometric grip force production, ${ }^{3}$ swallowing ${ }^{4}$ and the visualization of motor acts of teleological importance. ${ }^{5}$ While such studies have provided insight into the neural structures involved in the control of motor behaviors, they have not been able to probe the mechanisms of behavior acquisition, ${ }^{6}$ because there are currently no robotic, devices electromechanically suited for use in the high magnetic fields of a MRI scanner.

We outline a plan to design and develop a non-metallic, pneumatically actuated tool that, along with systems identification techniques and functional magnetic resonance imaging, will characterize and quantify how the human central nervous system uses sensory information during motor adaptation (practice-based motor learning). This knowledge is necessary to develop optimally effective therapies, technologies and interventions to improve motor function and independence in patients following stroke or other neural injury.

\section{SECTION II.}

\section{Methodology}

The development of a robotic device suitable for use in the challenging electromagnetic environment of an MRI scanner has progressed along the following stages:

1. Requirements Analysis: A detailed use case analysis has been performed to identify system requirements related to the material composition, mechanical performance, and noise tolerance of the robotic device and its controller.

2. Physical Modeling: Two physical models were Constructed to evaluate spatial envelope constraints.

3. Simulation: Computer simulations of the two prototype devices were performed to compare the mechanical responses to changes in air pressure and wrist angle. 
NOT THE PUBLISHED VERSION; this is the author's final, peer-reviewed manuscript. The published version may be accessed by following the link in the citation at the bottom of the page.

4. Validation and Verification: Testing was performed to ensure the device satisfied its specified requirements.

\section{SECTION III.}

\section{Results}

\section{A. Requirements Analysis}

Design of devices for use in the MRI environment must satisfy several material, size and noise tolerance limitations imposed by current MRI technologies. The high magnetic field generated by the MRI mandates the use of non-ferrous materials in all components of the design to ensure safety of the subject and the scanner. The device must also be small enough to fit inside the scanner with other instrumentation (visual display and EMG) without causing discomfort to the subject. It must provide a sufficient range of wrist movement (range: 20 degrees extension to 40 degrees flexion). The actuator must also produce sufficient force to perturb goal directed wrist movements ( $\sim$ 8-10 N), with an acceptable step response time and damping factor $(<0.2$ seconds and $>0.7$, respectively). Finally, movement of the upper torso and head must be eliminated to minimize motion artifact in the fMRI data. Consequently, our proposed device will measure and perturb flexion and extension movements of the wrist.

$\mathbf{A}^{\mathrm{k}}$

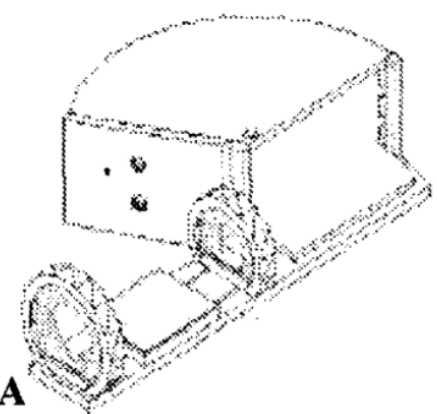

B

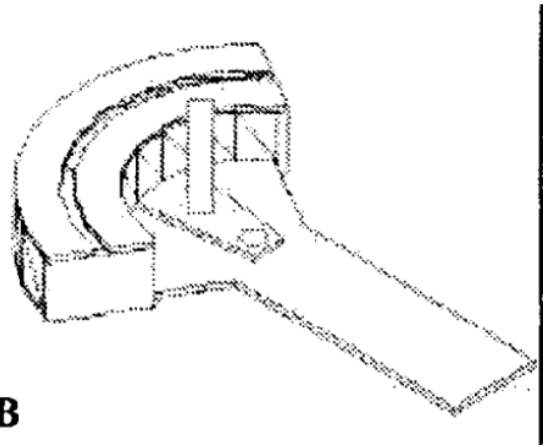

Figure 1 Two prototype models ( $a$ and $b$ ) of the wrist manipulandum.

\section{B. Physical Modeling}

Two prototype devices have been developed (Fig. 1). In both versions, the forearm of the subject is held tightly to the device by inflatable cuffs reducing motion of the subject's arm and ensuring proper positioning of their wrist and hand. Also, both systems operate by controlling the air pressure in a bladder trapped within an actuating volume. 
This bladder opposes motion in the flexion direction. The two prototypes are differentiated by how the hand interfaces to the device and by the air volume enclosed by the actuator. The subject's hand contacts a plate that is free to rotate about the wrist in the larger model (prototype A), while the hand grasps a handle in the smaller device (prototype B).

\section{Simulation}

The pneumatic actuator was simulated using Matlab and Simulink, The device was modeled as a variable capacitance bladder of infinite compliance that was trapped inside a rigid (but variable) volume with a sliding wall of negligible mass. Pressure in the variable volume obeyed the ideal gas law. The position of the sliding wall therefore depends on the balance of forces between subject-generated wrist torque and the product of the bladder pressure and the surface area of the sliding wall. The maximum torque the two systems can generate, assuming a driving pressure of $20 \mathrm{psi}$, is $\sim 325 \mathrm{Nm}$ and $\sim 60 \mathrm{Nm}$ for $\mathrm{A}$ and $\mathrm{B}$, respectively. The response of the two prototypes to a step change in commanded bladder pressure with a fixed volume was simulated (Fig. 2A). The step response time of the smaller device is more than 7 times faster than the larger one.

A second simulation evaluated the response of prototype $\mathbf{B}$ to a slow change in bladder volume when the commanded pressure is held constant As the volume approaches $0 \mathrm{cc}$, the pressure approaches infinity due to the inverse relationship between pressure and volume However, the response is approximately linear between 0 and 45 degrees wrist flexion, the anticipated operating range of the device. 

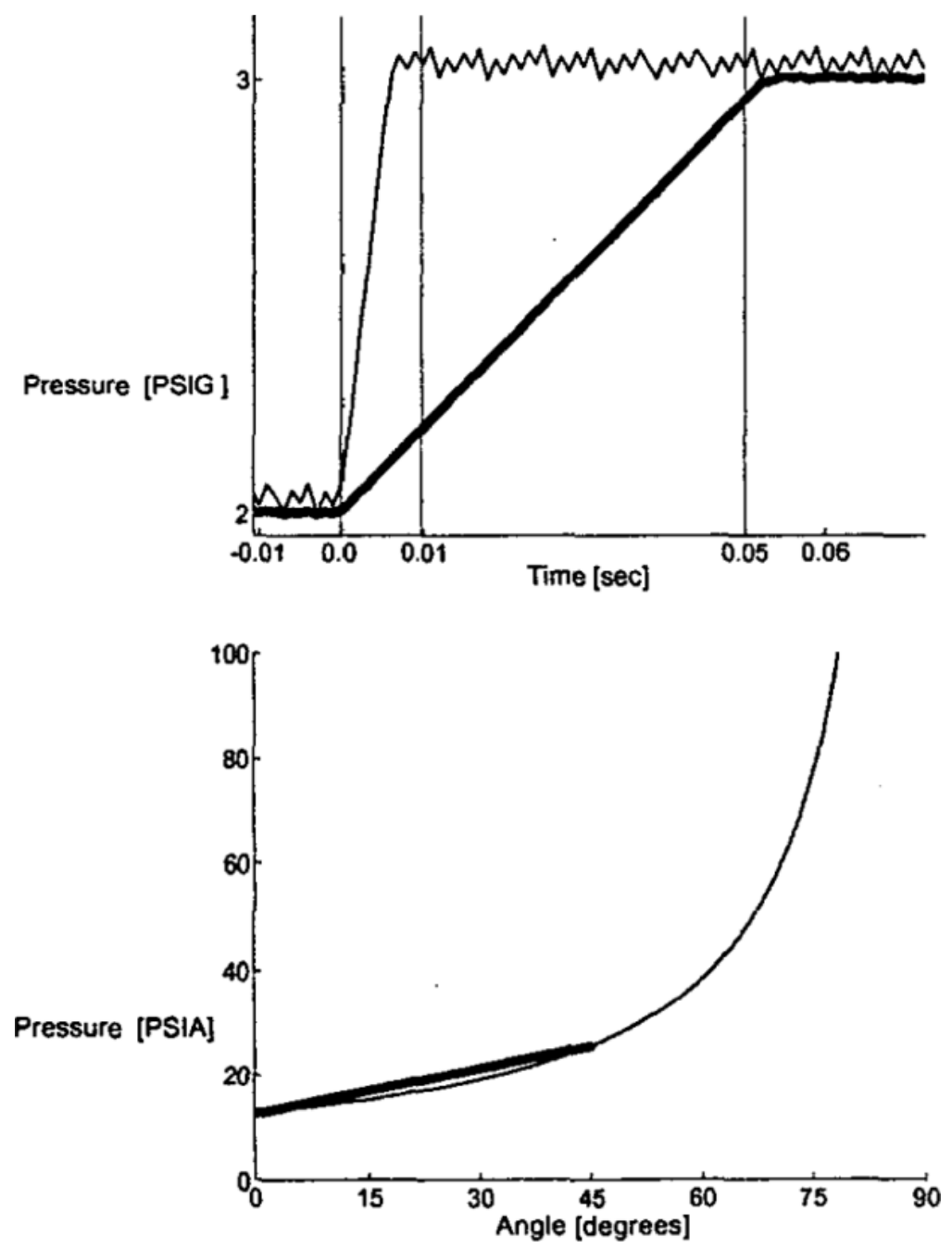

Figure 2 A) simulated step response of prototype a (thick line) and b (thin line)to a 1.0 psi change in driving pressure. $b$ ) simulated response of prototype $b$ as bladder volume changed from its maximum to its minimum value (thin line) the nearly-linear range is indcated by the dark solid line.

\section{Validation and Verification}

The device is currently being tested for conformance to the system requirements outlined above Preliminary testing of prototypes A and B in a mock MRI scanner identical to the scanner that will be used in this project was done to ensure that the size of the device was appropriate. Prototype A was clearly too large to accommodate the subject and auxiliary instrumentation, and therefore a redesign was necessary (prototype B). Testing of prototype $\mathbf{B}$ shows that its reduced size is sufficient to fit inside the MRI scanner with all necessary auxiliary instrumentation. 


\section{SECTION IV.}

\section{Discussion and Conclusion}

It is clear from the simulation result; that both devices are capable of generating more than adequate wrist torque, and that the smaller device has a much faster response time Initial experience in the MRI enxronment also indicates that a smaller device is better. Unfortunately, decreasing the size of the entrapped air volume (by decreasing the overall size of the device) causes the entrapped air pressure to vary in an increasingly nonlinear fashion to changes in wrist angle. This nonlinearity could complicate the design of a pneumatic control circuit, and is therefore considered undesirable. Therefore, the manipulandum resign poses a classic trade-off between size and performance. The device shown in Figure 1B represents a reasonable tradeoff between conflicting performance and size constraints.

\section{ACKNOWLEDGMENT}

This work is supported in part by a predoctoral GAANN fellowship from the Department of Education and by a grant from the Birnschein Foundation.

\section{References}

1E.R. Kandel, J.H. Schwartz, T.M. Jessell, Principles of Neural Science, McGraw-Hill: Health Professions, vol. 38, pp. 42-43, 2000.

2A.P. Georgopoulos, J.F. Kalaska, R. Caminiti, J.T. Massey, "Interruption of Motor Cortical Discharge Subserving Aimed Arm Movements", Exp. Brain Res., vol. 49, pp. 327-340, 1983.

${ }^{3}$ H.H. Ehrsson, A. Fagergren, H. Forssberg, "Differential Fronto-Parietal Activation Depending on Force Used in a Precision Grip Task: An fMRI Study", J. Neurophysiol., vol. 85, pp. 2613-2623, 2001.

4K Mosier, Liu W-C, J Maldjian, R Shah, B. Modi, "Lateralization of cortical function during swallowing: a fMRI study", AJNR Am J Neuroradiol, vol. 20, pp. 1520-1526, 1999.

5.A. Stevens, C. D. Mah, F. Mussa-Ivaldi, W.Z. Rymer, "Instantiation of an internal representation of a mechanical environment", Abstracts of the Society for the Neural Control of Movement Sevilla Spain, 2001.

6R. Shadmehr, F.A. Mussa-Ivaldi, "Adaptive representation of dynamics during learning of a motor task", J. Neurosci., vol. 14, pp. 3208-3224, 1994. 